\title{
Effect of Chemotherapy on Immune Factors in Patients with Non-Small Cell Lung Cancer
}

\author{
Jingjing Zhang1*, Dianbin Song2, Yi Dong1, Lu Bai', Dongqi Gao1, Shenglin Zhang1, Yan Guo1, \\ Fubo Li' ${ }^{1}$ Xiaolei Yu1, Qingshan Li1"
}

\author{
${ }^{1}$ Department of Oncology, The Affiliated Hospital of Chengde Medical University, Chengde, China \\ ${ }^{2}$ Department of Urology, The Affiliated Hospital of Chengde Medical University, Chengde, China \\ Email: 942636560@qq.com, "songdianbin123@163.com
}

How to cite this paper: Zhang, J.J., Song, D.B., Dong, Y., Bai, L., Gao, D.Q., Zhang, S.L., Guo, Y., Li, F.B., Yu, X.L. and Li, Q.S. (2021) Effect of Chemotherapy on Immune Factors in Patients with Non-Small Cell Lung Cancer. Journal of Biosciences and Medicines, 9, 110-117.

https://doi.org/10.4236/jbm.2021.912009

Received: November 17, 2021

Accepted: December 14, 2021

Published: December 17, 2021

Copyright $\odot 2021$ by author(s) and Scientific Research Publishing Inc. This work is licensed under the Creative Commons Attribution International License (CC BY 4.0).

http://creativecommons.org/licenses/by/4.0/

\begin{abstract}
Objective: To analyze the effects of different stages of chemotherapy on the immune cytokines (NKG2D, IL-12, IL-15, IL-18, DC cells, TNF-a, IFN-r) in patients with non-small cell lung cancer (NSCLC). Methods: 106 patients who met the research requirements in the Department of oncology of the Affiliated Hospital of Chengde Medical College from September 2018 to June 2021 were included in the study. The blood levels of interleukin-12 (IL-12), interleukin-15 (IL-15), interleukin-18 (IL-18), dendritic cells (DC cells), tumor necrosis factor A (TNF-a) and the levels of immune interferon (IFN-r) and NK cell activating receptor (NKG2D) in blood before chemotherapy, after the first chemotherapy and after the second chemotherapy were analyzed. Results: Except for the viability of DC cells and DC cells, all other immune factor groups showed statistical differences. Conclusion: Chemotherapy will have a negative effect on all immune factors except DC cells. The effect of immune factors will be weakened according to the increase of the chemotherapy cycle. Therefore, immunotherapy for non-small cell lung cancer needs to be carried out before chemotherapy or in the early stage of chemotherapy to achieve better results.
\end{abstract}

\section{Keywords}

Non-Small Cell Lung Cancer, Chemotherapy, Immune Cytokines

\section{Introduction}

With the improvement of the level of various medical examinations, the detection rate or incidence of malignant diseases has gradually increased, and the ${ }^{\star}$ First author.

\#Corresponding author. 
changes in the living environment have also changed the disease spectrum of malignant diseases. Some diseases that were rare in the past are now becoming common. The incidence of non-small cell lung cancer has increased in recent years, and it has become more and more advanced in the incidence of malignant tumors [1] [2].

With the gradual increase in the incidence of malignant tumors, the treatment of malignant tumors is also gradually innovating. Some malignant diseases may even be cured. Unfortunately, there is still no definite way to cure patients with lung cancer. But this does not prevent the research on lung cancer from becoming popular. At present, the most effective treatment for inhibiting the progression of lung cancer is still surgery or radiotherapy and chemotherapy, and some patients who have lost the opportunity of surgery can only be treated with radiotherapy and chemotherapy. Recently, immunotherapy for tumors has gradually emerged. After clinical application, a large number of documents have shown its effectiveness [3] [4]. Therefore, research on changes in immune factors during tumor treatment has also begun to become important.

As we all know, due to the extensive killing of chemotherapy, it will also cause serious damage to the body's immune system while killing tumor cells, killing a large number of cellular immune factors, but at the same time, it will also reversely stimulate the immune system due to the lack of cellular immune factors. A large number of immune factors, thereby partially improve the body's immune activity [5] [6] [7]. In response to this, we have studied the cellular immune factor activity in patients with non-small cell lung cancer (NSCLC) before and after chemotherapy.

\section{Object and Method}

\subsection{Basic Information}

We took NSCLC patients from the Oncology Department of the Affiliated Hospital of Chengde Medical College from September 2018 to June 2021 as the research object, Divided into control group, experimental group A, experimental group B, including 58 male patients and 48 female patients; the oldest is 78 years old, the youngest is 30 years old, and the average age is $(61.27 \pm 8.15)$ Years of age; Lung cancer classification: 26 patients with squamous cell carcinoma, 71 patients with adenocarcinoma, and 9 patients with adenosquamous carcinoma; TNM staging: 27 patients with stage III and 79 patients with stage IV.

Inclusion criteria: 1) Patients who were clearly diagnosed as NSCLC by pathology. 2) Patients between 18 - 80 years old. 3) Patients who have lost the opportunity for surgery after the assessment of the expertise in surgery, imaging, and pathology. 4) KPS score $\geq 60$ points. 5) Patients whose survival period exceeds 3 months. 6) No chemotherapy contraindications.

Exclusion criteria: 1) Patients with surgical indications requiring surgery. 2) Combined with serious basic diseases, such as immune system, severe dysfunction of head, heart, lungs and abdominal organs, severe infection, blood system 
diseases, etc.

All research subjects were approved by the ethics committee of the Affiliated Hospital of Chengde Medical College and were enrolled and signed an informed consent form.

\subsection{Chemotherapy}

The chemotherapy regimen is cisplatin/carboplatin + pemetrexed/gemcitabine/ paclitaxel, and the dosage: pemetrexed $500 \mathrm{mg} / \mathrm{m}^{2}$, gemcitabine $1250 \mathrm{mg} / \mathrm{m}^{2}$, paclitaxel $175-200 \mathrm{mg} / \mathrm{m}^{2}$, cisplatin $75 \mathrm{mg} / \mathrm{m}^{2}$, carboplatin AUC $=6$, intravenous drip administration, 21 days as a cycle [8].

\subsection{Research Methods}

All patients received $30 \mathrm{ml}$ of upper limb venous peripheral blood on an empty stomach in the morning before chemotherapy on the day of chemotherapy, after chemotherapy on the 22nd day of the first chemotherapy, and after chemotherapy on the 22nd day of the second chemotherapy. Spin/separate the core for 20 minutes (centrifugation radius $14 \mathrm{~cm}$ ), take the supernatant and place it in a refrigerator at $-80^{\circ} \mathrm{C}$ for later use. Enzyme-linked immunosorbent assay was used to detect the levels of NKG2D, IL-12, IL-15, IL-18, DC cells, DC cell viability, TNF-a, IFN-r in serum. All factor detection kits were purchased from Kangtaihe Yuan Biotechnology Co.Ltd. (Beijing).

\section{Statistical Analysis}

The statistical analysis of the study was performed using SPSS20.0 software. Count test uses $\mathrm{t}$ test, measurement data uses $\chi^{2}$ test, test standard $\alpha=0.05, \mathrm{P}<\alpha$ has statistical significance (Table 1).

\section{Discussion}

With the gradual evolution of the spectrum of malignant tumors, lung cancer is now the disease with the highest incidence among malignant tumors, and its mortality rate is also the first [9]. The high mortality rate is mainly due to the limitation of treatment methods. The cure rate of lung cancer is currently less than $20 \%$, and the cure rate of advanced lung cancer that loses the chance of surgery is even less than 5\% [10] [11]. The most common of many types of lung cancer is non-small cell lung cancer (NSCLC), and because of its strong invisibility, it is not easy to find in the early stage. Generally, it has reached the middle and advanced stage when it is discovered. Many patients have lost the opportunity to operate. The research on the treatment of NSCLC is currently mainly focused on chemotherapy [12].

NSCLC chemotherapy regimens are mainly platinum-based dual combination drugs, but all the research reports on chemotherapy regimens for this disease so far point out that the treatment effect is not ideal, and the huge side effects brought by chemotherapy make some patients intolerable had to give up treatment [13]. 
Table 1. Comparison of the expression of each factor between the experimental group and the control group.

\begin{tabular}{|c|c|c|c|c|c|}
\hline Immunocytokine & Control group & $\begin{array}{c}\text { Experiment } \\
\text { Group A }\end{array}$ & $\begin{array}{c}\text { Experiment } \\
\text { Group B }\end{array}$ & $\mathrm{t}$ value & $P$ value \\
\hline IFN-r & $1.27 \pm 0.314$ & $0.85 \pm 0.20$ & & 7.209 & 0.001 \\
\hline IFN-r & $1.27 \pm 0.314$ & & $0.39 \pm 0.192$ & 16.011 & 0.001 \\
\hline IFN-r & & $0.85 \pm 0.20$ & $0.39 \pm 0.192$ & 11.547 & 0.001 \\
\hline IL-12 & $10.07 \pm 1.259$ & $8.52 \pm 1.078$ & & 6.899 & 0.001 \\
\hline IL-12 & $10.07 \pm 1.259$ & & $6.28 \pm 1.183$ & 15.604 & 0.001 \\
\hline IL-12 & & $8.52 \pm 1.078$ & $6.28 \pm 1.183$ & 9.703 & 0.001 \\
\hline IL-15 & $4.40 \pm 1.091$ & $3.29 \pm 1.170$ & & 4.834 & 0.001 \\
\hline IL-15 & $4.40 \pm 1.091$ & & $1.97 \pm 0.886$ & 12.064 & 0.001 \\
\hline IL-15 & & $3.29 \pm 1.170$ & $1.97 \pm 0.886$ & 6.244 & 0.001 \\
\hline IL-18 & $138.27 \pm 9.265$ & $92.08 \pm 7.960$ & & 25.068 & 0.001 \\
\hline IL-18 & $138.27 \pm 9.265$ & & $59.58 \pm 6.742$ & 47.583 & 0.001 \\
\hline IL-18 & & $92.08 \pm 7.960$ & $59.58 \pm 6.742$ & 22.913 & 0.001 \\
\hline DC cell & $1.47 \pm 0.339$ & $1.51 \pm 0.214$ & & 1.231 & 0.222 \\
\hline DC cell & $1.47 \pm .339$ & & $1.55 \pm 0.212$ & 0.387 & 0.700 \\
\hline DC cell & & $1.51 \pm 0.214$ & $1.55 \pm .212$ & -0.930 & 0.355 \\
\hline DC Cell viability & $0.98 \pm 0.012$ & $0.98 \pm 0.013$ & & -0.472 & 0.638 \\
\hline DC Cell viability & $0.98 \pm 0.012$ & & $0.98 \pm 0.090$ & -1.510 & 0.135 \\
\hline DC Cell viability & & $0.98 \pm 0.013$ & $0.98 \pm 0.090$ & -0891 & 0.375 \\
\hline TNF-a & $8.09 \pm 0.748$ & $5.57 \pm .644$ & & 16.035 & 0.001 \\
\hline TNF-a & $8.09 \pm 0.748$ & & $3.33 \pm 0.646$ & 30.566 & 0.001 \\
\hline TNF-a & & $5.57 \pm .644$ & $3.33 \pm 0.646$ & 79.007 & 0.001 \\
\hline NKG2D & $2.02 \pm 0.252$ & $1.65 \pm .127$ & & 8.727 & 0.001 \\
\hline NKG2D & $2.02 \pm 0.252$ & & $1.45 \pm 0.136$ & 13.197 & 0.001 \\
\hline NKG2D & & $1.65 \pm .127$ & $1.45 \pm 0.136$ & 7.279 & 0.001 \\
\hline
\end{tabular}

Focus: Except for the viability of DC cells and DC cells, there are statistical differences between the experimental group and the control group and the experimental two groups of other factors.

With the rapid development of medicine at present, with the gradual deepening of tumor research, tumor-related diseases and immune-related research are also gradually being carried out, and there is a lot of positive news. Immunotherapy mainly achieves the purpose of treatment by enhancing the body's own immune function, that is, increasing the expression number or expression activity of various immune factors in the body [14]. However, current research points out that immunotherapy alone cannot achieve the desired therapeutic effect. It can only be achieved through immunotherapy and adjuvant chemotherapy. Therefore, at present, immunotherapy combined with chemotherapy has become a 
popular research direction for tumor treatment [15]. However, the indiscriminateness of chemotherapeutic drugs has increased the uncertainty of combination therapy. Therefore, finding the right time to increase immunotherapy during chemotherapy is the key to achieving better results of combination therapy.

Studies have found that in human immunity, T lymphocytes, NK cells, dendritic cells and other immune cells play an important role in human anti-tumor immunity, and their related receptors or immune factors such as NKG2D, IL-12, IL-15, The number or activity of IL-18, DC cells, TNF-a, and IFN-r can indirectly indicate the strength of the body's immune function [16] [17] [18].

NKG2D is an important receptor of NK cells, and it can activate NK cells after binding to the corresponding ligands, allowing NK cells to exert their anti-tumor effects. Therefore, the expression level of NKG2D largely determines the size of the anti-tumor effects of NK cells, and partly determines the strength of the human immune function [19]. IL-12 is a cytokine that is relatively beneficial to the human body. It can promote the expression of most immune cells, such as DC cells, NK cells, and macrophages. It can also stimulate such as IFN- $\gamma$ to increase NK cells, The secretion of immune factors with $\mathrm{T}$ cell activity, and the above effects have laid an important foundation for IL-12 in the human body's anti-tumor effect [20]. IL-15 is a cytokine with functions similar to IL-12 discovered in the past 20 years. It can not only directly stimulate the expression of immune cells but also stimulate the secretion of related immune factors acting on immune cells, so as to achieve anti-tumor effects [21]. IL-18 is a cytokine that can act on most immune cells. Due to its wide range of biological activities, some studies have shown that IL-18 can also act on tumor cells to regulate tumor cell proliferation, occurrence, development and even Inhibition and growth of related blood vessels [22]. Dendritic cells (DC) are the most powerful antigenpresenting cells in the human body, and differentiated mature DC cells are the only antigen-presenting cells that can induce the activation of naive T lymphocytes, so DC cells are also Play an important role in the immune system [23]. TNF-a and IFN-r are both important cytokines in the anti-tumor process, with high-efficiency and extensive immunomodulatory effects.

The above factors are involved in the regulation of the body's immune function, and play an important anti-tumor effect for patients with malignant tumors. In this study, we compared the factors before and after chemotherapy, and found that all factors except DC cells decreased significantly after chemotherapy, and the activity of each factor decreased with the increase of the chemotherapy cycle, indicating that chemotherapy significantly inhibited the body's immune function. It also partially explains that in the process of chemotherapy combined with immunotherapy, it is necessary to increase immunotherapy before chemotherapy, increase the activity of various immune factors, and enhance the body's immunity to counter the immunosuppressive effects of chemotherapy and increase the efficacy of combined therapy. In the next step, we will conduct correlation research on the types of different factors to clarify the relationship between different factors, so as to further refine the different immune stimulating 
effects of immunotherapy, and more targeted combination therapy. The disadvantage of this study is that the enrolled patients come from a single center, and the chemotherapy cycle is still short, which may cause bias in the results. In the future, the sample size and the study cycle should be expanded to confirm this conclusion.

\section{Conclusion}

Chemotherapy will have a negative effect on all immune factors except DC cells. The effect of immune factors will be weakened according to the increase of the chemotherapy cycle. Therefore, immunotherapy for non-small cell lung cancer needs to be carried out before chemotherapy or in the early stage of chemotherapy to achieve better satisfaction.

\section{Fund Project}

S\&T Program of Chengde (201804a030).

\section{Conflicts of Interest}

The authors declare no conflicts of interest regarding the publication of this paper.

\section{References}

[1] Du, X., Shao, Y., Qin, H.F. and Gao, H.J. (2018) ALK-Rearrangement in NonSmall-Cell Lung Cancer (NSCLC). Thoracic Cancer, 9, 423-430. https://doi.org/10.1111/1759-7714.12613

[2] Samaraweera, L., Adomako, A., Rodriguez-Gabin, A. and McDaid, H.M. (2017) A Novel Indication for Panobinostat as a Senolytic Drug in NSCLC and HNSCC. Scientific Reports, 7, Article No. 1900. https://doi.org/10.1038/s41598-017-01964-1

[3] Proto, C., Ferrara, R., Signorelli, D., Lo Russo, G., Galli, G., Imbimbo, M., et al. (2019) Choosing Wisely First Line Immunotherapy in Non-Small Cell Lung Cancer (NSCLC): What to Add and What to Leave Out. Cancer Treatment Reviews, 75, 39-51. https://doi.org/10.1016/j.ctrv.2019.03.004

[4] Ferrer, L., Levra, M.G., Brevet, M., Antoine, M., Mazieres, J., Rossi, G., et al. (2019) A Brief Report of Transformation from NSCLC to SCLC: Molecular and Therapeutic Characteristics. Journal of Thoracic Oncology, 14, 130-134. https://doi.org/10.1016/j.jtho.2018.08.2028

[5] Camidge, D.R., Peters, S., Mok, T., Gadgeel, S.M., Cheema, P.K., Pavlakis, N., et al. (2018) Updated Efficacy and Safety Data from the Global Phase III ALEX Study of Alectinib (AL) versus Crizotinib (CZ) in Untreated Advanced ALK+ NSCLC. Journal of Clinical Oncology, 36, 9043. https://doi.org/10.1200/JCO.2018.36.15 suppl.9043

[6] Girard, N. (2018) Optimizing Outcomes in EGFR Mutation-Positive NSCLC: Which Tyrosine Kinase Inhibitor and When? Future Oncology, 14, 1117-1132. https://doi.org/10.2217/fon-2017-0636

[7] Wang, T., Wang, X., Du, Q., Wu, N., Liu, X., Chen, Y., et al. (2019) The CircRNA CircP4HB Promotes NSCLC Aggressiveness and Metastasis by Sponging miR-133a5p. Biochemical and Biophysical Research Communications, 513, 904-911. 
https://doi.org/10.1016/j.bbrc.2019.04.108

[8] Tan, A.C. (2020) Targeting the PI3K/Akt/mTOR Pathway in Non-Small Cell Lung Cancer (NSCLC). Thoracic Cancer, 11, 511-518. https://doi.org/10.1111/1759-7714.13328

[9] Mayo-de-Las-Casas, C., Jordana-Ariza, N., Garzón-Ibañez, M., Balada-Bel, A., BertránAlamillo, J., Viteri-Ramírez, S., et al. (2017) Large Scale, Prospective Screening of EGFR Mutations in the Blood of Advanced NSCLC Patients to Guide Treatment Decisions. Annals of Oncology, 28, 2248-2255. https://doi.org/10.1093/annonc/mdx288

[10] Zhu, X., Chen, L., Liu, L. and Niu, X. (2019) EMT-Mediated Acquired EGFR-TKI Resistance in NSCLC: Mechanisms and Strategies. Frontiers in Oncology, 9, Article No. 1044. https://doi.org/10.3389/fonc.2019.01044

[11] Remon, J., Caramella, C., Jovelet, C., Lacroix, L., Lawson, A., Smalley, S., et al. (2017) Osimertinib Benefit In EGFR-Mutant NSCLC Patients with T790M-Mutation Detected by Circulating Tumour DNA. Annals of Oncology, 28, 784-790. https://doi.org/10.1093/annonc/mdx017

[12] Dagogo-Jack, I., Rooney, M., Nagy, R.J., Lin, J.J., Chin, E., Ferris, L.A., et al. (2019) Molecular Analysis of Plasma from Patients with ROS1-Positive NSCLC. Journal of Thoracic Oncology, 14, 816-824. https://doi.org/10.1016/j.jtho.2019.01.009

[13] Garinet, S., Laurent-Puig, P., Blons, H. and Oudart, J.B. (2018) Current and Future Molecular Testing in NSCLC, What Can We Expect from New Sequencing Technologies? Journal of Clinical Medicine, 7, Article No. 144.

https://doi.org/10.3390/jcm7060144

[14] Russo, A.E., Priolo, D., Antonelli, G., Libra, M., Mccubrey, J.A. and Ferraù, F. (2017) Bevacizumab in the Treatment of NSCLC: Patient Selection and Perspectives. Lung Cancer. Targets and Therapy, 8, 259-269. https://doi.org/10.2147/LCTT.S110306

[15] Papadimitrakopoulou, V., Cobo, M., Bordoni, R., et al. (2018) IMpower132: PFS and Safety Results with 1L Atezolizumab+ Carboplatin/Cisplatin+ Pemetrexed in Stage IV Non-Squamous NSCLC. Journal of Thoracic Oncology, 13, S332-S333. https://doi.org/10.1016/j.jtho.2018.08.262

[16] Frank, R., Scheffler, M., Merkelbach-Bruse, S., et al. (2018) Clinical and Pathological Characteristics of KEAP1- and NFE2L2-Mutated Non-Small Cell Lung Carcinoma (NSCLC). Clinical Cancer Research, 24, 3087-3096. https://doi.org/10.1158/1078-0432.CCR-17-3416

[17] Nakamura, R., Inage, Y., Tobita, R., Yoneyama, S., Numata, T., Ota, K., et al. (2018) Sarcopenia in Resected NSCLC: Effect on Postoperative Outcomes. Journal of Thoracic Oncology, 13, 895-903. https://doi.org/10.1016/j.jtho.2018.04.035

[18] Lee, D.H. (2017) Treatments for EGFR-Mutant Non-Small Cell Lung Cancer (NSCLC): The Road to a Success, Paved with Failures. Pharmacology \& Therapeutics, 174, 1-21. https://doi.org/10.1016/j.pharmthera.2017.02.001

[19] Li, Y., Hu, J., Li, L., Cai, S., Zhang, H., Zhu, X., et al. (2018) Upregulated Circular RNA circ_0016760 Indicates Unfavorable Prognosis in NSCLC and Promotes Cell Progression through miR-1287/GAGE1 Axis. Biochemical and Biophysical Research Communications, 503, 2089-2094. https://doi.org/10.1016/j.bbrc.2018.07.164

[20] Le, X., Puri, S., Negrao, M.V., Nilsson, M.B., Robichaux, J., Boyle, T., et al. (2018) Landscape of EGFR-Dependent and-Independent Resistance Mechanisms to Osimertinib and Continuation Therapy beyond Progression in EGFR-Mutant NSCLC. Clinical Cancer Research, 24, 6195-6203. https://doi.org/10.1158/1078-0432.CCR-18-1542 
[21] Santaniello, A., Napolitano, F., Servetto, A., De Placido, P., Silvestris, N., Bianco, C., et al. (2019) Tumour Microenvironment and Immune Evasion in EGFR Addicted NSCLC: Hurdles and Possibilities. Cancers, 11, Article No. 1419.

https://doi.org/10.3390/cancers11101419

[22] Lisberg, A., Tucker, D.A., Goldman, J.W., Wolf, B., Carroll, J., Hardy, A., et al. (2018) Treatment-Related Adverse Events Predict Improved Clinical Outcome in NSCLC Patients on KEYNOTE-001 at a Single Center. Cancer Immunology Research, 6, 288-294. https://doi.org/10.1158/2326-6066.CIR-17-0063

[23] Volckmar, A.L., Leichsenring, J., Kirchner, M., Christopoulos, P., Neumann, O., Budczies, J., et al. (2019) Combined Targeted DNA and RNA Sequencing of Advanced NSCLC in Routine Molecular Diagnostics: Analysis of the First 3,000 Heidelberg Cases. International Journal of Cancer, 145, 649-661.

https://doi.org/10.1002/ijc.32133 